\title{
Incidence of Knee Osteoarthritis after Anterior Cruciate Ligament Injury- A Systematic Review
}

\author{
Sami S. Almureef ${ }^{1}$ \\ Senior Physiotherapist Prince SultanMilitary Medical City -Riyadh Saudi Arabia
}

\begin{abstract}
Background: This is a systematic review of studies on the prevalence and risk factors of knee osteoarthritis more than four years following anterior cruciate ligament tear.

Methods: A systematic search was performed in PubMed, EMBASE and AMED. Inclusion criteria were studies involving patients with isolated anterior cruciate ligament injury or combined with other knee meniscal or ligaments injuries treated by operative or non- operative methods and assessed by radiological clinical evaluation tools and radiological classification systems. Coleman modified score was used to assess the methodological quality of the included studies.

Results: Five prospective, three of which were randomised controlled trail, and three retrospective studies were included. All studies were of good quality with Coleman modified score higher than 55. The mean score was 76 prevalence of knee OA following ACL injuries, varying between 0\% and 59\%. Meniscal injury and duration between injury and surgery were found to be the most common risk factors for knee OA following ACL injury.

Conclusion: This systematic review of good quality studies suggests that the prevalence rates of knee osteoarthritis after anterior cruciate ligament reconstruction varies between 0 to 59\% Patients with combined ACL and meniscal injury. There was wide variation in the reported knee OA prevalence. These variations are attributed to factors such as differences in study population, study design, participant's level of activity, reconstruction approaches, post-operative rehabilitation programme and radiological classification used. Overall, the modified Coleman methodology score was high for the included studies. Only one study included non-operatively treated patients making comparison between the outcome of operative and non-operative approach difficult.
\end{abstract}

Keywords: anterior cruciate ligament injury; knee osteoarthritis; mid-to long-term follow- up

\section{Introduction}

The ACL is a strong ligament connecting the femur to the tibia. Anatomically, it has two bundles, the Antero-medial, which originates from the proximal part of the femoral insertion to the antero-medial part of the tibial insertion. This bundle becomes loaded and stretched during knee flexion, and the poster-lateral bundle inserts into the postero-lateral part of the tibial plateau which gets loaded and stretched during knee extension1.The main function of the ACL is to support and stabilise the tibia from anterior displacement relative to the femur as well as stabilise internal and external rotation, valgus and varus movements of the tibia over the femur2. ACL injury is one of the most common injuries to ligaments of the knee and accounts for approximately 30 injuries per 100,000 of the population3. However, there are more than 100,000 new ACL injuries that happen each year4 (Agel et al., 2005). ACL is a common injury for those playing sport and among the general public. It is more common in the 15-25 age group4, particularly those involved in pivoting sports like football, tennis. Seventy percent of ACL injuries happen in non-contact situations when the player lands with external rotation of the tibia in relation to the femur with the knee fully extended5. Research shows that women are at higher risk than men4,6.

In people with ACL injuries, knee function impairment might be demonstrated in the form of limited ROM, muscle weakness and pain leading to limitation of daily living or sports activities7. One of the most reported consequences of an ACL injury is lack of dynamic knee stability, where stability of the joint is not held during fast changes of position during open and closed chain activities8.Quadriceps are some of the most affected muscles following an ACL injury and reconstruction9. ACL Reconstruction has a range detrimental effect on joint function such as: reduced muscle function, reduced ROM and neuromuscular deficits10. Joint effusion is found to be associated with reduced knee function as well11

The long term effect of ACL reconstruction and rehabilitation on knee function has been discussed extensively in the literature. 12Deehan conducted a case series study, which demonstrated the 15 year outcome of ACL reconstruction with emphasis on knee functions for 90 patients who had isolated ACL injury. The study used a good range of outcome measures including ROM, Lachman and pivot shift tests, single leg hop test, lysholm knee score, radiographic assessment and International Knee Documentation Committee evaluation. Study results 
indicated good ligament stability, ROM and subjective outcome measures at 15 years after surgery with kneeling pain as a common persistent problem. The radiological assessment has indicated signs of OA, although the severity of these signs was not associated with the level of joint stability. 13Daniel conducted a prospective study to investigate the fate of 292 patients who had ACL injuries. These patients were treated either surgically or non-surgically. The study results indicated that in the five-year followup all the participants who were treated by non-surgical approaches returned to normal sports activities, and those who underwent reconstruction developed a higher level of OA compared with the non-operated patients. ACL Injuries can be clinically treated by conservative rehabilitation or surgical repair followed by post-surgical rehabilitation. The clinical decision regarding which treatment pathway is usually made by the surgeon and the patient14. Conservative, on-surgical, rehabilitation aims to restore the joint dynamic stability and function, through strength and neuromuscular training2. Quadriceps weakness is evident after anterior cruciate ligament (ACL) injury, which might affect the knee function post operatively15. Knee osteoarthritis (KOA) is one of the major causes of pain and physical disability in older adults. This is a dynamic disease that is metabolically active and includes both destruction and construction mechanisms that could be activated by injuries from either biochemical or mechanical events1. No denitive treatment was found to be superior and the patho-mechanics are still not well understood 1,16 . There are a range of structural changes associated with knee OA, which include damage or loss of articular cartilage, remodelling and scelrosis of the subchondral bone, subchondral cysts formation and osteophyte formation 17.

\section{Materials And Methods}

Aims of the study

- To review the reported prevalence of knee OA after ACL injuries treated with conservative treatment or surgery with a follow-up more than four years.

- To evaluate the reported risk factors for knee OA following ACL injury.

Literature search

\begin{tabular}{|l|l|l|}
\hline S.N & Table 1 : Search Terms & \\
\hline 1 & Anterior cruciate ligament & 13259 \\
\hline 2 & Knee joint & 62929 \\
\hline 3 & Knee Joint OR ligament & 108585 \\
\hline 4 & Knee injuries & 24488 \\
\hline 5 & Osteoarthritis & 53943 \\
\hline 6 & Epidemiologic studies & 1574831 \\
\hline 7 & Epidemiology & 1612945 \\
\hline 8 & Retrospective studies & 481967 \\
\hline 9 & 2 OR 5 & 104397 \\
\hline 10 & 1 Or 3 Or 4 & 77474 \\
\hline 11 & 6 Or 7 & 2701369 \\
\hline 12 & 9 AND 10 AND 11 & 2896 \\
\hline
\end{tabular}

Table 1: Search Terms

Systematic searches were carried out in the following databases: PubMed, Medline, CINHAL, AMED, OVID, Cochrane Library and PEDRO, from the inception of the databases to October 2013. MeSH terms and Boolean operators were used for journal articles related to ACL and osteoarthritis. The searches were conducted by the primary researcher. The search strategy and specific search terms used are explained in table (1). The abstracts of the search results were reviewed by the main researcher to identify the studies that match the inclusion criteria. If the information given in the abstract was not sufficient to make a decision, the full text version of the article was reviewed.

\section{Design:}

Research methodology as the path to finding an answer to a research question18. Systematic review of the literature in this study attempts to answer the research question by systematically evaluating related literature. It summarises their results in order to identify the prevalence of knee Osteoarthritis following ACL injuries. Over 20,000 journals and two million articles are published every year. This increasing amount of available papers does not make it easy for health professionals to conclude clinical facts and recommendations. Therefore, systematic review is considered to be at the highest level of the evidence hierarchy 19 as it offers the researcher opportunity to contribute to the literature via exploring the available studies related to the topic of interest and conclude some evidence that could be applied in clinical practice 20. The aim of the PRISMA Statement is to assist researchers with improving the reporting of systematic reviews and meta- analyses 21 . A critical literature review is a valuable scientific method to summarise, critically appraise and communicate the 
results of available research22. Therefore, it is helpful in pooling together results from separately conducted research related to the prevalence of KOA following ACL injuries. In addition to the previous attributes, systematic reviews are important to identify gaps in the current literature and guide future research activities 23 .

\section{Inclusion Criteria}

The inclusion criteria were prospective or retrospective studies in English or translated into English, published between 1960 and 2013. Studies might include male or female adult subjects with ACL injury and treated surgically by any type of technique or using any type of graft or treated non- surgically by rehabilitation programme. The follow-up time was more than four years.

\section{Exclusion criteria}

The exclusion criteria were not prospective or retrospective case studies, and articles that reported individuals with ACL injury associated with fractures. These exclusion criteria were established to minimize the clinical heterogeneity of the review outcomes..

\section{Search results:}

Figure 1 shows the results from the search strategy and the application of the PRISMA flow diagram24. A total of 2896 studies were identified using the search strategy from the inception of the databases until October 2013. After removing duplicates, 1831 studies were screened against the inclusion criteria, 85 full text articles were assessed for eligibility and finally 39 studies were identified. Studies that were included.25 were excluded to avoid duplication and finally four studies were assessed as prospective and three studies were assessed as retrospective.

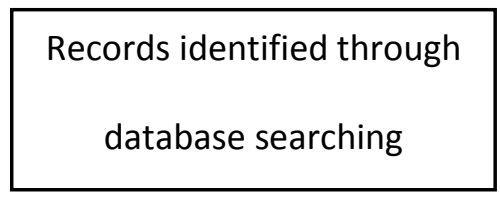

Full text articles screened for eligibility $(n=85)$

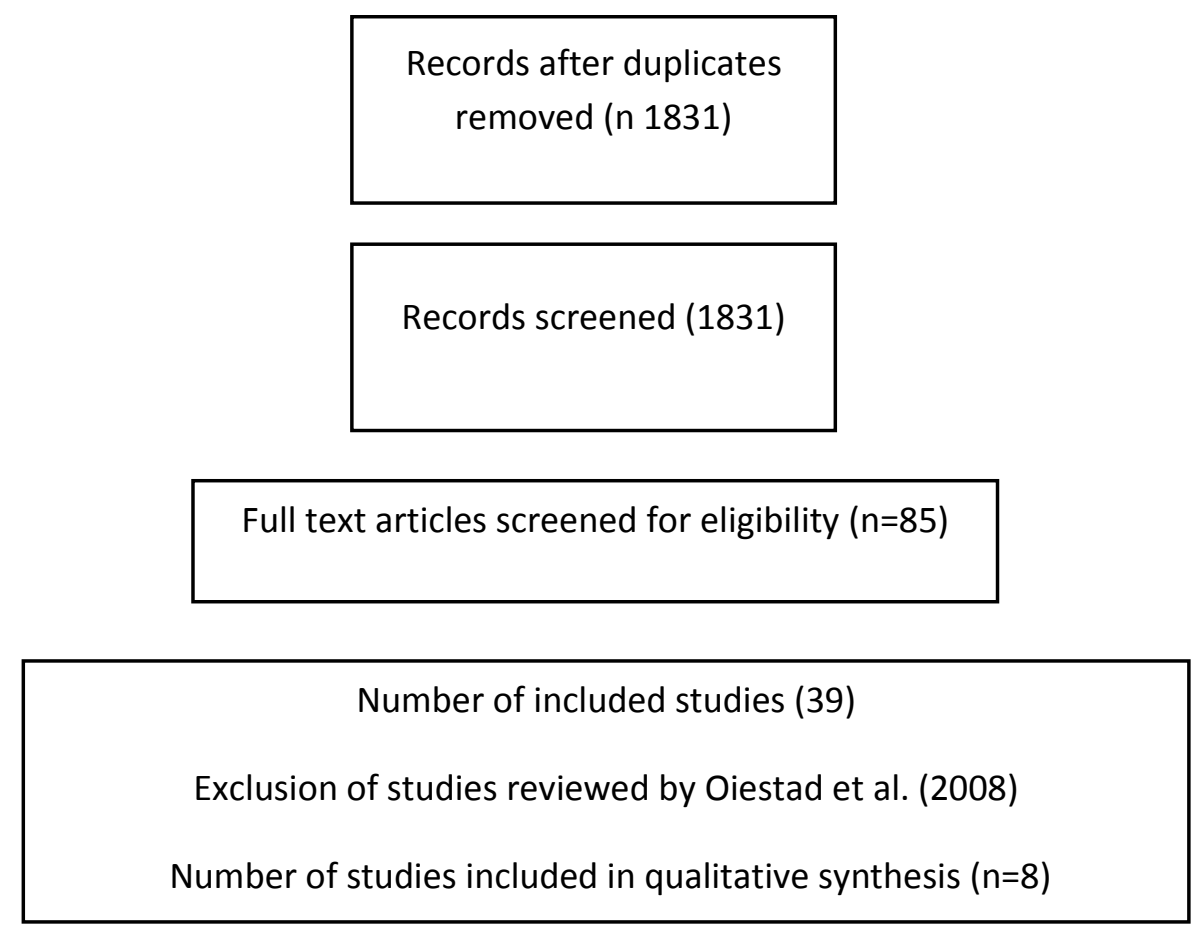

Additional records identified through other sniurres $(n=28)$

Figure 1 
Data collection and analysis: Data was read and collected by the main reviewer using data extractions form (see table 2).

Table 2Characteristics of Studies included in the Systematic Review

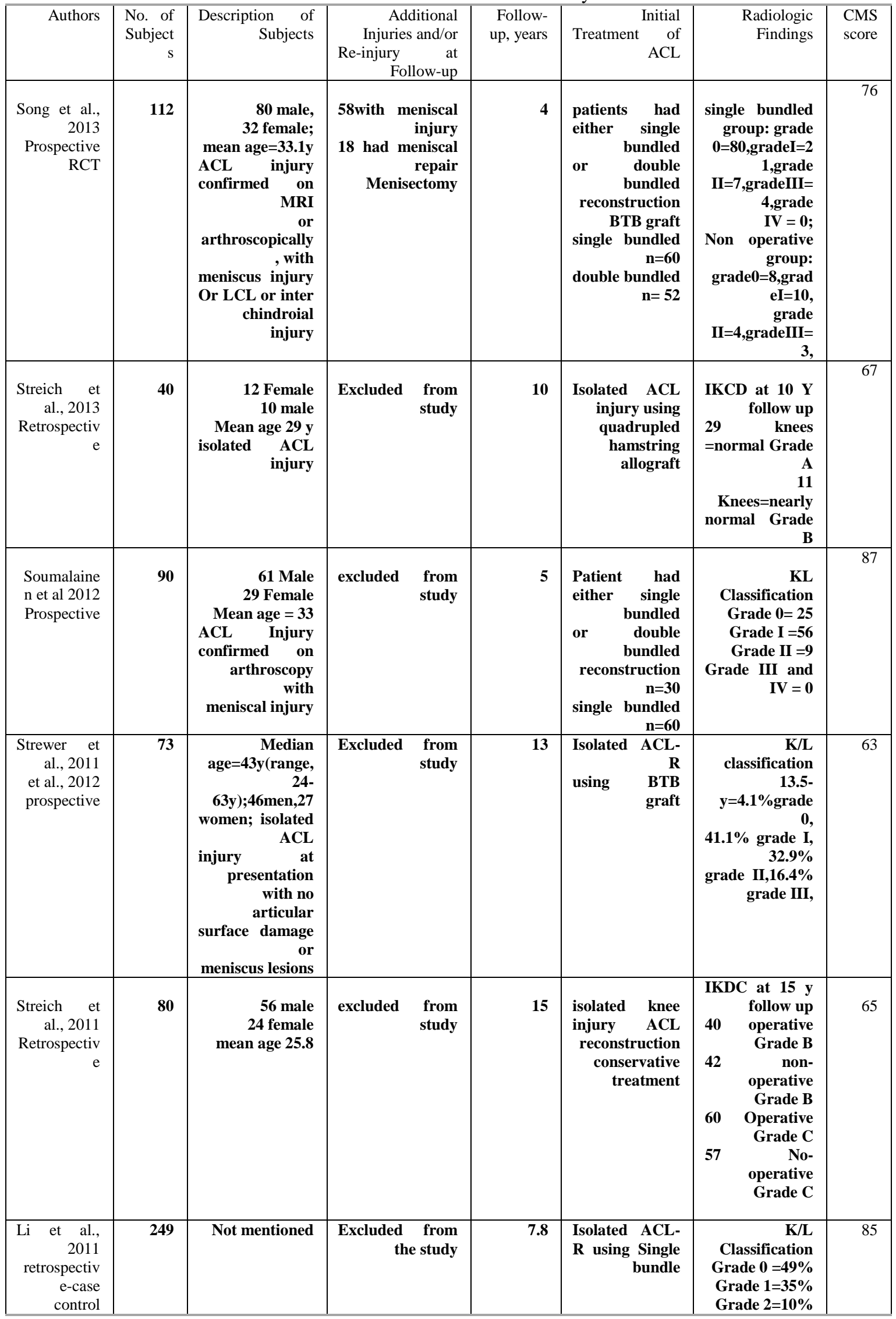




\begin{tabular}{|c|c|c|c|c|c|c|c|}
\hline & & & & & & Grade $4=2 \%$ & \\
\hline $\begin{array}{r}\text { Oiestad et } \\
\text { al., } 2010 \\
\text { Prospective }\end{array}$ & 221 & $\begin{array}{r}\text { 90 females, 120 } \\
\text { males; mean } \\
\text { age=39.1 } \\
\text { isolated ACL } \\
\text { or in } \\
\text { in } \\
\text { combination } \\
\text { with meniscus } \\
\text { injury and/or } \\
\text { chrondral lesion } \\
\text { and/or } \\
\text { injury, } \\
\text { wCL with } \\
\text { uninjured } \\
\text { contralateral } \\
\text { knee }\end{array}$ & 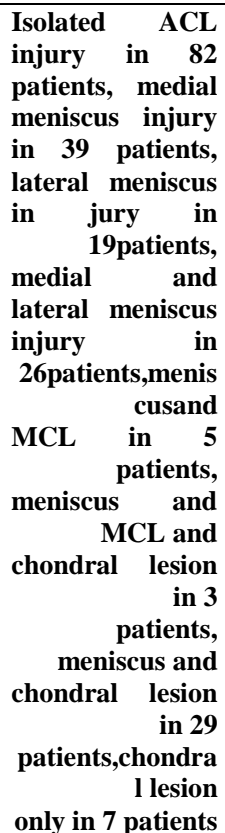 & 12.5 & 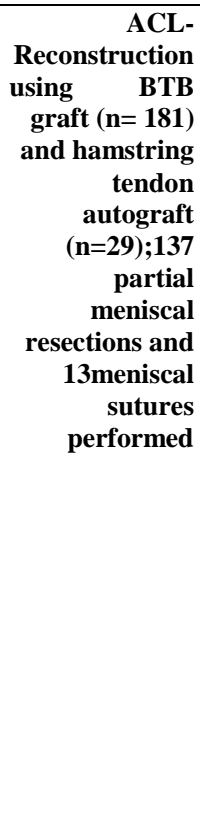 & 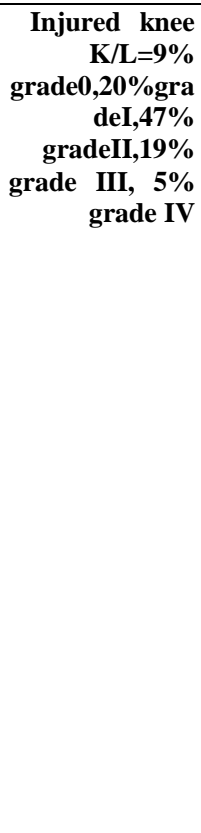 & 87 \\
\hline $\begin{array}{r}\text { Keays et al., } \\
2010 \\
\text { Prospective }\end{array}$ & 56 & $\begin{array}{r}18 \text { Female } \\
40 \text { Male } \\
\text { Mean age } 27 \text { y } \\
\text { isolated ACL or } \\
\text { in combination } \\
\text { withmensical or } \\
\text { chondral injury. }\end{array}$ & $\begin{array}{r}\text { Isolated ACL or } \\
\text { combined } \\
\text { with } \begin{array}{r}\text { meniscal } \\
\text { injury }\end{array}\end{array}$ & & $\begin{array}{r}\text { ACL- } \\
\text { Reconstruction } \\
\text { using BTB } \\
\text { graft }(\mathbf{n}=29) \\
\text { and hamstring } \\
\text { tendon } \\
\text { autograft } \\
(n=29)\end{array}$ & $\begin{array}{r}\mathrm{K} / \mathrm{L}= \\
\operatorname{grad} \mathrm{I}=48 \% \\
12 \% \text { grade II, } \\
36 \% \text { grade III }\end{array}$ & 67 \\
\hline
\end{tabular}

Review of the literature:

\section{Result}

Overview of the studies

Studies were collected from PubMed, Medline, CINHAL, AMED, OVID, Cochrane Library and PEDRO databases. All studies were published in peer reviewed journals, e.g. American Journal of Sport Medicine and Physiotherapy. Eight studies were included in this review. The studies investigated the prevalence and risk factors for knee osteoarthritis following isolated ACL or combined with meniscal or chondral lesions. 921 subjects were included in this review with sample size ranging from 40 to 249 subjects with mean age of 25.8 to 39.The patients had either isolated ACL injury ( $\mathrm{n}=614$ subjects) or ACL and associated injuries $(\mathrm{n}=307$ subjects). ACL and associated injury were evaluated by clinical and radiologic methods or using both methods. The studies were conducted in the USA, Australia or European countries. All the studies used radiological assessment to evaluate KOA at the follow-up. One study included the radiological findings of 40 subjects who received non-operative treatment; seven studiesincluded radiological assessment of 841 patients treated surgically; and three studies included radiological assessment of 40 subjects treated non-surgically and surgically 26.

There were 881 patients treated surgically by different surgical approaches. Bone-patellar tendon-bone grafts were performed on 395 subjects, 98 subjects were surgically treated using hamstring tendon graft and 288 were treated by combination of both grafts. One study used single bundled and double bundled grafts. Forty patients were treated by non-operative methods 26 . This included physiotherapy and quadriceps and/or lower limb strengthening for a minimum of three months and or lifestyle modification instructions.

Methodological Quality

The results of the study quality assessments are presented in table 3 .None of the studies scored the complete mark of 90 and fulfilled all the criteria. The highest score was 87 , achieved 30 , and the lowest score was 6526.The prospective studies achieved a mean modified CMS of 76, with a highest score of 87 and a lowest score of 67. The retrospective studies achieved a mean score of 72, with a highest score of 65 and a lowest score of 85 . Studies that achieved scores above 55\% were considered of high quality 27. 


\section{Table 3}

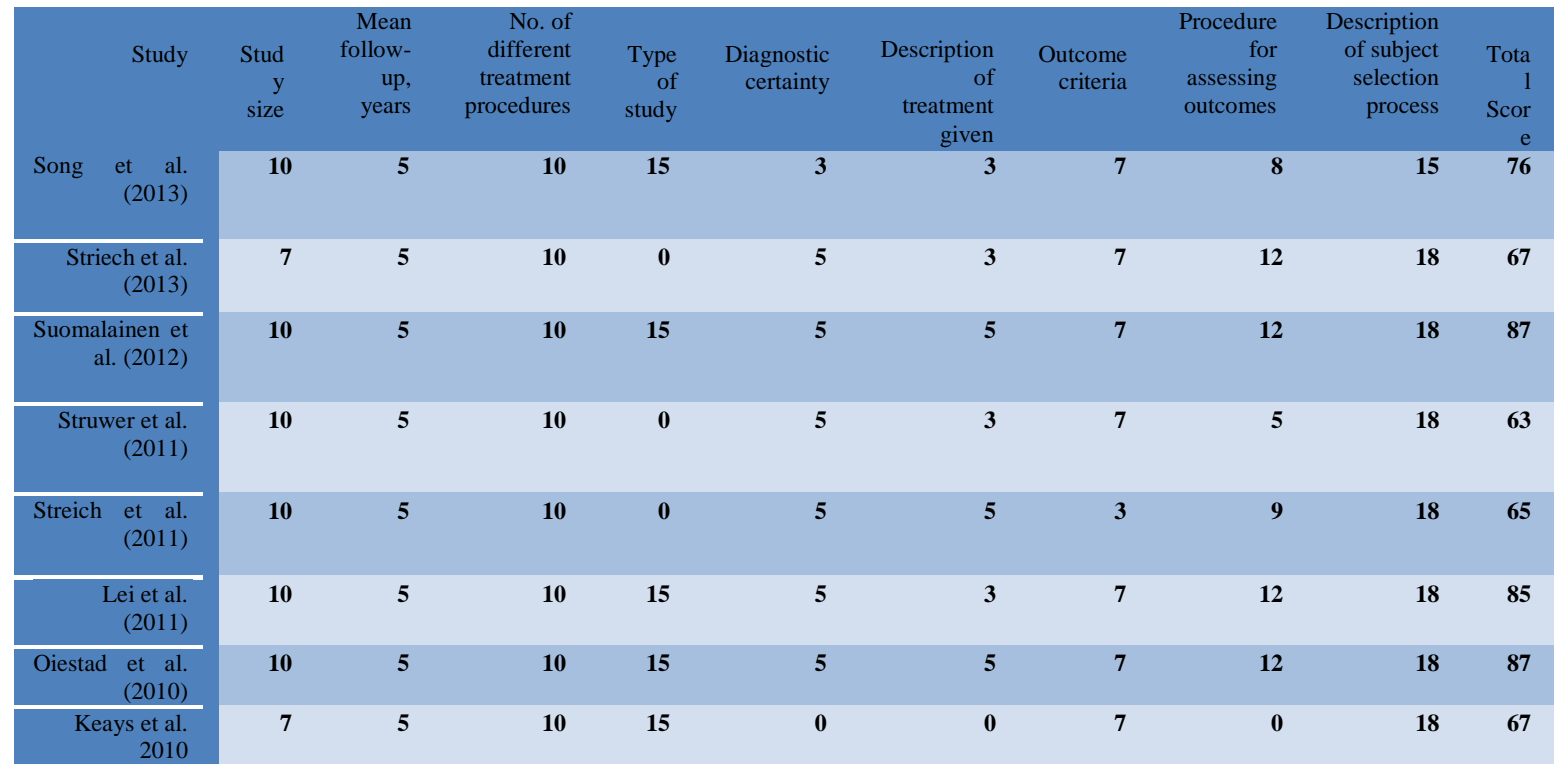

Table 4Risk Factors for Development of Knee OA following ACL rupture

\begin{tabular}{l|l|}
$\begin{array}{l}\text { Study used Discriminate } \\
\text { analysis }\end{array}$ & Strong Risk Factors \\
\hline Striech et al (2013) & Menisectomy-meniscal injury-duration between injury and surgery-BMI -Age \\
\hline Keays et al 2010 & Menisectomy-meniscal injury-duration between injury and surgery \\
\hline
\end{tabular}

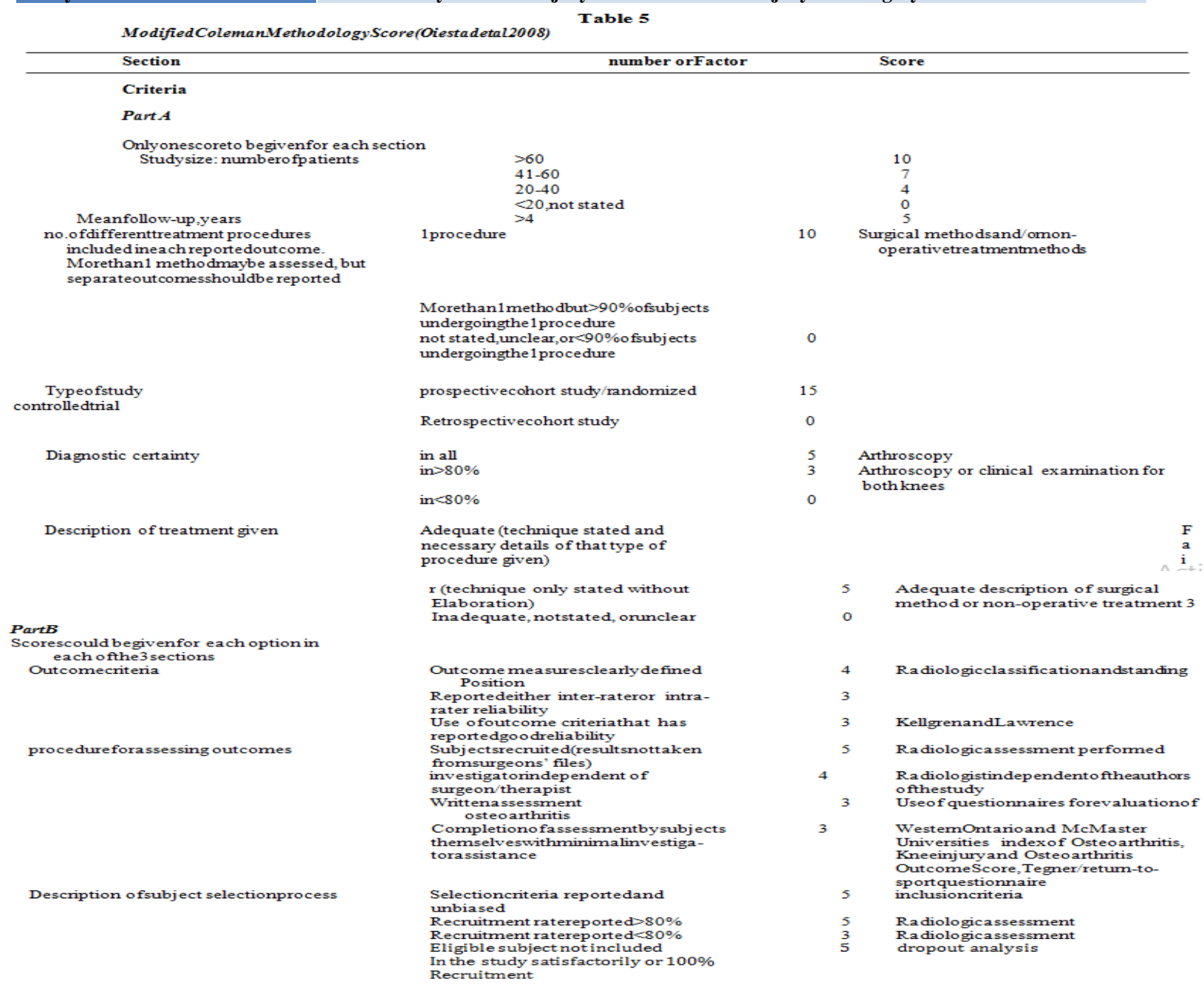


The Critical Appraisal Skills Programme 10 Guide Questions (Table 5) was used to explore the strengths and weaknesses of a research paper. In the present literature review, each paper is analysed individually. The initial step in analysing a research is critiquing the aim or purpose of the study 28.

All the included studies demonstrated a very clear and well formulated title. It is important that a study has a well- defined title to attract more readers and give them a clear idea of the main objectives29. This could on occur if the title describes the study design, sample population, time and duration of the study. Observational study design is considered the most frequently used research method to observe prevalence and risk factors ofpathologies30. Most of the follow-up studies on subjects with ACL injuries were either prospective or retrospective cohort studies with few RCTs as well. Cohort studies are characterised by observation of interventions, exposures or outcome over a certain period as they occur naturally from the time of inclusion 31 . A case-control design also includes observation of a control group without the exposure of interest. There are different factors that might affect the validity of cohort studies. This includes selection bias, confounding factors and possibility of high drop rate of participants at the follow-up. A common methodological bias issue related to long-term follow-up studies is the follow-up rate of the study participants. Follow-up rate might be affected by the difficulties of finding the study participants at the time of follow-up, participants' readiness and motivation to participate as well as cost related issue particularly for those who live a long distance away.

32this depends on the type of loss, whether it is systematic or random, i.e. considerable bias might be taken into account if there is a systematic loss of participants to follow-up of $20 \%$. On the other hand, random loss to follow-up of up to $60 \%$ has been shown to give unbiased results. These figures might be different with RCTs in which $20 \%$ loss at follow-up or more is considered a real threat to the study validity 33 . The prospective cohort study design is the most adequate design for evaluating long-term knee function and development of knee OA in subjects with ACL injury and reconstruction.

Song et al., 2013

This prospective randomised controlled trail (RCT) study investigated whether double bundled ACL reconstruction is superior to single bundled technique to prevent OA or provide better stability and function. The study recruited 130 patients with unilateral ACL injury and no instability or previous radiological evidence of KOA (more than grade 2 Kellgren and Lawrance classification). The participants were prospectively randomised into a double bundled $(n=65)$ or single bundled $(n=65)$ group. Degree of osteoarthritis was evaluated according to the Kellgren-Lawrance grade prior to the surgery and at the time of the follow-up four years later. The number of patients with one degree or more progression of $\mathrm{OA}$ from pre to post operation was determined. Lachman and pivot-shift tests were used to evaluate stability and Lysholm knee score, Tegner activity score and International Knee Documentation Committee (IKDC) subjective scale were used to evaluate the functional outcomes. Using a combination of reliable subjective scales and objective measures is essential to evaluate improvement in functional activities9. Six patients dropped at the time of the follow-up due to graft failure and had revision surgery. This drop rate is not big enough to produce bias33. Five patients (9.6\%) in the double bundled group and six patients (10\%) in the single bundled group showed more advanced OA radiological changes at the follow-up. Within six month of surgery, all patients regained full range of knee movement and no significant differences in stability were found. Also, functional scales did not show significant difference between groups in terms of changes in functional activities except for International knee Documentation Committee where $(\mathrm{P}=0.03)$. The time from injury to surgery, age at surgery and sex follow-up periods were not significantly different between the two groups and not considered as risk factors of KOA in this study. However, patients who underwent meniscetomy or meniscal repair showed more degenerative changes compared with those who did not $(\mathrm{P}=0.04)$. The authors clearly stated the aim of the study and established the rationale of conducting this research. They explored the available evidence that supports the prevalence of KOA following single bundled ACL reconstruction due to the post-surgical changes in knee kinematics. Considering the evidence from anatomical studies that suggest better knee kinematics following double bundled techniques, and the lack of randomised trial to investigate prevalence of KOA following the double bundled technique, the authors have used this as a basis to conduct the trial.

\section{Streich et al., 2013}

This retrospective study investigated the long-term outcome of ACL reconstruction using quadrupled semitendinosus tendon. The rationale behind the study was clearly explained by the authors. Patellar tendon bone allograft is widely used in ACL reconstruction, although it has several complications such as high incidence of anterior knee pain, quadriceps weakness and limited range of knee extension. Therefore, hamstring tendon allograft is becoming more popular. However, short- and long-term studies have indicated several postoperative complications associated with hamstring tendon allograft too, including hamstring weakness and increased joint laxity. That said, no studies have been conducted to investigate the long-term effect of hamstring tendon allograft using the quadrupled graft, which is stronger and bigger in size and might provide better results. There is need for such a study. In this study, 101 patients were diagnosed by clinical examination and confirmed 
with arthroscopy, which improved the validity of the diagnosis. The patients underwent ACL reconstruction using the quadrupled hamstring graft, and those with chondral, ligamentous or meniscal injuries were excluded. This was helpful to produce a homogenous sample. In total, 59 patients participated. There was a random drop rate of 19 patients due to various reasons (the details of the dropped patients are mentioned by the authors. Details of the surgical procedure were clearly set out to help reproducibility. However, all surgeries were performed by one senior surgeon, and no details were given about surgeon experience. Final assessment at 10 years follow-up period was performed by two independent blinded observers to minimize bias. The assessment was conducted using radiographic examination following the International Knee Documentation Committee guidelines. 34Valley considered this classification as valid and reliable classification to assess knee degenerative changes. Lysholm and Tegner activity scores were used for clinical evaluation. Although the study has used the contralateral knee for comparison at the end of the follow-up period, it is not clear whether initial assessment was preoperatively conducted. This might raise questions regarding how improvement or deterioration could be accurately detected and might affect the validity of the study. The study mentioned what was permitted and when during the rehabilitation process, but no details were given about the rehabilitation setting, components of the exercise programme or the physiotherapists who supervised the rehabilitation programme. This seems to be an issue with all the previous studies and might negatively affect the reproducibility.

Statistical analysis and results were clearly discussed using graphs. Study results showed that 11 patients had mild evidence of degeneration and graded as B by the International Knee Committee Documentation. However, the rest of the sample was graded as A, which is normal. No patient was classified as grade $\mathrm{C}$ or $\mathrm{D}$, which is defined as severely abnormal by the International Knee Committee Documentation guidelines. The study suggested negative correlation between pivot shift and clinical assessment score (IKDC and Lyshom scores), which is in line with previous findings and earlier research by the authors of this study 35. Patients with negative pivot test had better results on IKDC and lyshom scores and those with signs of rotational instability. The study also showed positive correlation between age and High BMI index and knee degenerative changes. Overall, the study was the first to investigate the long-term outcome of ACL reconstruction using quadrupled hamstring graft, although some issues might affect the reproducibility and generalisability of the study such as small sample size, methodological flaws related to lack of details regarding pre-operative clinical assessment, limited details of the rehabilitation process and surgeon experience. However, it opens the door for future research to be conducted using larger sample size.

\section{Summary of the literature review}

All the studies demonstrated aim and objectives based on sound justification. No sample size calculation was performed in any of the included studies. Therefore, there was unjustifiable variation in sample size; number of subjects varied between 40and 24936. The source of sample was stated in all the studies as well as inclusion and exclusion criteria. All studies mentioned the number of patients lost at follow-up and explained the causes of the drop rate at final follow-up. Description of the sample population was clear to help with the reproducibility of the study method in most of the studies. However, the details provided varied, with most of the studies giving a detailed description of the sample sex and mean age. Others provided further details about patient level of activities. Additionally, the studies used a range of reliable clinical and radiographic outcomes. Some studies used a wide range of measures 37 , while others used one measure to assess each aspect of the clinical examination37. All the studies clearly described the surgical procedures to improve reproducibility. However, all the studies except that of failed to provide information on surgeon experience38. Details of the post-operative rehabilitation programme consistently lacked details in all the studies except for Oiestad. Details of assessor blinding were considered in, but this was not considered in any other study39. Five studies used prospective study design, which was considered by a previous review as the most appropriate to investigate prevalence of OA following ACL rupture.This study design is superior to the retrospective design. The chances of recall bias and loss of participants at follow-up is less compared with retrospective study design 40 Overall, the studies demonstrated different levels of methodological quality. A common issue which may have affected the reproducibility of these studies was limited detail as regards some parts of the methods such as postoperative rehabilitation, assessment procedures and lack of assessor blinding.

This chapter summarised important points that included an overview of the main characteristics of the studies, the results of assessing the risk bias using the Modified Coleman Methodology Score and the methodological quality, outcomes and recommendations of each study.

\section{Summary of the main results}

\section{Discussion}

Eight studies of good quality (Coleman Modified score CMS > 55) including a total of 921 subjects with ACL injury were included in this systematic review based on the inclusion criteria. Five of the studies were assessed to have a prospective study design, and three studies were assessed to have a retrospective study 
design. The studies achieved a mean Coleman Modified score (CMS) of 74.6 of a total score of 90 . The prospective studies disclosed higher methodological quality than did the retrospective studies. Mean modified CMS for the prospective studies was 76 and 47 for the retrospective studies.

In this review two different radiological classifications were used by the studies: International Knee Documentation Committee and Kellgren-Lawrance classification. In studies which used International Knee Documentation Committee grade (A), there was no evidence of radiological degeneration and grade D indicated severe degeneration. In studies which used Kellgren and Lawrence classification, grade 0 indicated no evidence of radiologic classification and grade IV indicated severe degeneration. To maintain consistency, Grade B in the International Knee Documentation Committee and Grade II in the Kellgren and Lawrence classification were considered as the cut-off grade to indicate degeneration at the follow-up evaluation41.

The reported prevalence of knee OA in this review varied between $0 \%$ and $59 \%$. Five of the studies included subjects with isolated knee injuries and showed prevalence of knee OA of 0\% (Soumalainen CMS score of 87 ) to $49 \%$ (Streich - SMS score of 65 ). Studies with subjects combining ACL and meniscal injury showed prevalence of knee OA which varied between 0\% (Streich et al. 2013 - CMS score of 67) and 42.3\% (Oiestad CMS score of 87).

The study which included subjects with isolated knee injury and achieved second highest SCM score reported knee OA prevalence of $0 \% 44$. In comparison, the study with the highest CMS score and included subjects with combined ACL and meniscal injury reported a knee OA prevalence of $42.3 \%$. In summary, the study results indicated that isolated ACL injury have low prevalence of radiologic knee OA compared with those with combined ACL and meniscal injury.Only one study with CMS score of 65 compared subjects treated by operative and non-operative approaches. The study reported no significant difference between the two groups with regard to the prevalence of knee OA.

The review of these results shows a wide range of variations in the reported knee OA prevalence. These variations might be attributed to different factors such as study population, study design, participant's level of activity, reconstruction approaches, post-operative rehabilitation programme and radiological classification used. These factors were considered by previous review. However, this review included more prospective studies; three were randomised controlled trials and the mean CMS score was higher than that of the study by the CMS score for the included studies in this review was relatively higher than those included.

One prospective case control study with CMS of 6742 and one retrospective study with CMS score of $85 \%$ used regression analysis to identify risk factors for development of knee OA following ACL injury. Different risk factors were reported by these two studies; the strongest factors were concurrent meniscal injury, duration between injury and surgery and Body Mass Index (BMI). A previous review considered the same factors as strong predictors as well.

Although previous studies have highlighted the positive impact of post-operative rehabilitation on the outcome of ACL reconstruction 43, most of the included studies did not highlight the possible effect of lack of supervised rehabilitation on the long-term clinical outcome. Future studies need to provide participants with comprehensive rehabilitation exercise programme in order to confirm post-operative rehabilitation as a possible risk factor for development of knee OA.

\section{Implication of the study}

The results of this study, which indicate low to moderate prevalence of knee OA following ACL reconstruction, support the findings of previous reviews. In line with previous studies, this review reported meniscal injury and time between injury to surgery as strong predictors of knee OA following ACL injury. The implication of these results on the timing of surgical approach, surgery performed early after the incidence of ACL rupture, might prevent further damage to the meniscus and articular cartilage and, consequently, reduce the risk of arthritis and prevent reoccurrence of rupture. In addition, such immediate surgical interference will reduce the age at surgery and the risk of patello-femoral OA.

\section{Strengths of the review}

One of the key strengths of this review is the inclusion of five prospective studies, three of which are randomised controlled trials. All studies including the three retrospective cohort studies scored over 55 in the Coleman modified score, which indicates good quality. Inclusion of good quality studies is important to reduce the biased outcomes 44 .

\section{Limitations of the review}

The study has a few limitations that might affect its validity. The data search was conducted by the researcher; unfortunately, it was difficult due to time constraint to use an independent librarian to conduct the data search to ensure that all the relevant studies were included. Although the researcher took all the necessary measures to identify the relevant literature, as a novice researcher there is possibility of error or inaccuracy in the data search. 
The researcher expanded the electronic search to cover the period from the inception of the included databases to October 2013, in order to identify any literature not included in the previous reviews. The screening of this large number of abstracts and main articles was a time consuming process. The researcher felt at the time that this would be helpful to improve the validity of the study. The search results identified the same studies included in the previous review. The researcher considers this as a beneficial learning experience and good attempt to improve the validity of the review.Due to time limitation, only one reviewer conducted the data extraction and quality scoring processes; this might affect the validity of the review due to increased risk of reviewer bias45.In this review a qualitative (narrative) analysis was conducted to analyse the findings of the studies and quality score. This method of analysis is considered to be subjective in nature as it reflects the reviewer's interpretation of the results, particularly in the absence of a peer reviewer, due to lack of statistical analysis. There were many variations among the studies which affected the data analysis process. Heterogeneity of the studies due to variable study design, assessment tools and population used in the trials made it difficult to conduct a quantitative analysis (meta-analysis) in addition to the narrative analysis in this review.

These variations in age and other variables in individual studies might lead to statistically heterogeneous outcomes. Clinical and statistical heterogeneity are closely related to each other46.Considering that ACL injury is more linked to sports related activities, patients with higher level of motivation and desire to return to sport early might be more willing to participate in such studies. Therefore, this might produce evidence of selection bias 47.

\section{Recommendations for future study}

Based on the results of this study, it is recommended that further prospective studies are conducted using less variables such as outcome measures, previous level of activity and age. Existence of such good quality trials will produce good quality meta-analysis studies. Such high level evidence is essential to guide change in clinical practice. Future studies might use regression analysis to investigate the risk factors for developing knee $\mathrm{O}$ following ACL rupture. Although risk factor was a common aim among the studies in this review, only two studies used regression analysis to produce valid results.

\section{Conclusion}

In conclusion, based on the quality assessment of the included studies and narrative analysis, the prevalence of knee OA was found to vary between 0 and $58 \%$ in this review. The highest prevalence was $49 \%$ reported by a retrospective study with Coleman modified score of $87 \%$. Lowest prevalence was 0 and achieved by a prospective study with Coleman modified score of $85 \%$.Different risk factors were considered as predictors of knee OA following ACL injury. Among these factors, meniscal injury, time between injury and surgery and BMI were found to be the strongest predictors. This review supports the evidence that the prevalence of knee OA following ACL injury is under 50\% as described by a mixture of good quality prospective and retrospective studies. Future research might need to be prospective and pay more attention to the importance of post- operative rehabilitation and other variables that could lead to heterogeneity of the studies such as population and level of activities.

\section{References}

[1] Petersen, W. \&Zantop, T. (2007). Anatomy of the anterior cruciate ligament with regard to its two bundles. Clinical orthopaedics and related research, 454, 35-47.

[2] Beynnon, B.D., Johnson, R.J., Abate, J.A., Fleming, B.C. \& Nichols, C.E.( 2005). Treatment of anterior cruciate ligament injuries, part $1 \& 2$ The American journal of sports medicine, 33(11), 1751-1767.

[3] Ageberg, E., Thomeé, R., Neeter, C., Silbernagel, K.G., \&Roos, E.M.( 2008). Muscle strength and functional performance in patients with anterior cruciate ligament injury treated with training and surgical reconstruction or training only: a two to five-year follow-up. Arthritis Care \& Research, 59(12), 1773-1779.

[4] Agel, J., Arendt, E.A. ,\&Bershadsky, B. (2005). Anterior cruciate ligament injury in national collegiate athletic association basketball and soccer: a 13-year review. The American journal of sports medicine, 33(4), 524-530

[5] Krosshaug, T., Nakamae, A., Boden, B.P., Engebretsen, L., Smith, G., Slauterbeck, J.R., Hewett, T.E. \& Bahr, R. (2007). Mechanisms of anterior cruciate ligament injury in basketball: video analysis of 39 cases. The American journal of sports medicine, 35(3), 359-367.

[6] Risberg, M.A., Holm, I., Steen, H., Eriksson, J. \&Ekeland, A. (1999). The effect of knee bracing after anterior cruciate ligament reconstruction. A prospective, randomized study with two years' follow-up. The American journal of sports medicine, 27(1), 76-83.

[7] Tegner, Y., Gustafsson, B., Forssblad, M., Lundgren, L., \&Sölveborn, S. A. (2007). Brain concussion and sports--new guidelines for management]. Läkartidningen, 104(16), 1220.

[8] Rudolph, K.S., Axe, M.J., Buchanan, T.S., Scholz, J.P. \& Snyder-Mackler, L. (2001). Dynamic stability in the anterior cruciate ligament deficient knee. Knee Surgery, Sports Traumatology, Arthroscopy, 9(2), 62-71.

[9] Segal, N.A., Glass, N.A., Torner, J., Yang, M., Felson, D.T., Sharma, L., Nevitt, M. \& Lewis, C.E. (2010). Quadriceps weakness predicts risk for knee joint space narrowing in women in the MOST cohort. Osteoarthritis and Cartilage, 18(6), 769-775.

[10] Kartus, J., Movin, T. \&Karlsson, J. (2001). Donor-site morbidity and anterior knee problems after anterior cruciate ligament reconstruction using autografts. The Journal of Arthroscopic \& Related Surgery, 17(9), 971- 980.

[11] Palmieri-Smith, R.M., Thomas, A.C. \&Wojtys, E.M. (2008). Maximizing quadriceps strength after ACL reconstruction. Clinics in sports medicine, 27(3), 405-424.

[12] Deehan, D.J., Salmon, L.J., Webb, V.J., Davies, A. \&Pinczewski, L.A. (2000). Endoscopic reconstruction of the anterior cruciate

[13] ligament with an ipsilateral patellar tendon autograft. A prospective longitudinal five-year study. Journal of Bone \& Joint Surgery, [14] British Volume, 82(7), 984-991.

[15] Daniel, D.M., Stone, M.L., Dobson, B.E., Fithian, D.C., Rossman, D.J. \& Kaufman, K.R. (1994). Fate of the ACL-injured patient. A prospective outcome study. The American journal of sports medicine, 22(5), 632-644.

[16] Altman, D. G., Schulz, K. F., Moher, D., Egger, M., Davidoff, F., Elbourne, D., Gotzsche, P.C., Lang, T. \& Trials, C. G. C. S. o. R. (2001). The revised CONSORT statement for reporting randomized trials: explanation and elaboration. Annals of internal medicine, 134(8),663-694. 
[17] Logerstedt, D., Lynch, A., Axe, M.J. \& Snyder-Mackler, L. (2013). Pre-operative quadriceps strength predicts IKDC2000 scores 6 months after anterior cruciate ligament reconstruction. The Knee, 20(3), 208- 212.

[18] Stevens, K.J. \&Dragoo, J.L. (2006).Anterior cruciate ligament tears and associated injuries. Topics in Magnetic Resonance Imaging, 17(5), 347-362.

[19] Buckwalter, J.A. \& Martin, J.A. (2006). Osteoarthritis. Advanced drug delivery review, 58(2):150-167.

[20] Kumar, R. (2005). Research Methodology: A Step-by-step Guide for Beginners. (2nd ed.)London: SAGE Publications Ltd.

[21] Evans, D. (2003). Hierarchy of evidence: a framework for ranking evidence evaluating healthcare interventions Journal of clinical nursing, 12(1), 77-84.

[22] Lang, T.A. (2004). The value of systematic reviews as research activities in medical education. Academic Medicine, 79(11), 1067-

[23] 1072

[24] Liberati, A., Altman, D.G., Tetzlaff, J., Mulrow, C., Gøtzsche, P.C., Ioannidis, J.P., Clarke, M., Devereaux, P.J., Kleijnen, J. \&Moher, D.

[25] (2009). The PRISMA statement for reporting systematic reviews and meta- analyses of studies that evaluate health care interventions: explanation and elaboration. Annals of internal medicine, 151(4), 264-269.

[26] Spendlove, D. (2008). Creativity in education: a review. Design and Technology Education: An International Journal, 10(2).

[27] Kitchenham, B. (2004). Procedures for performing systematic reviews. Keele, UK, Keele University,33, 2004.

[28] Moher, D., Liberati, A., Tetzlaff, J., Altman, D.G. \& Group, P.( 2009). Preferred reporting items for systematic reviews and metaanalyses: the PRISMA statement. Annals of internal medicine, 151(4), 264-269.

[29] Oiestad, B.E., Engebretsen, L., Storheim, K. \&Risberg, M.A. (2009). Knee osteoarthritis after anterior cruciate ligament injury: a

[30] systematic review. The American journal of sports medicine, 37(7), 1434-1443.

[31] Streich, N.A., Zimmermann, D., Bode, G. \& Schmitt, H.(2011). Reconstructive versus non-reconstructive treatment of anterior [32] cruciate ligament insufficiency. A retrospective matched-pair long-term follow- up. International orthopaedics, 35(4), 607-613.

[33] Van Tulder, M.W., Touray, T., Furlan, A.D., Solway, S., Bouter, L.M. \& Group, C.B.R.(2003). Muscle relaxants for nonspecific low back pain: a systematic review within the framework of the cochrane collaboration. Spine, 28(17), 1978-1992.

[34] Stockhausen, L., \&Conrick, M. (2002). Making sense of research: a guide for critiquing a paper. Contemporary nurse, 14(1), 38-48.

[35] Babor, M., Gerzon, S., Raveh, B., Sobolev, V., \& Edelman, M. (2008). Prediction of transition metal- binding sites from apo protein structures. Proteins: Structure, Function, and Bioinformatics, 70(1), 208-217.

[36] Bhandari, M., Morshed, S., Tornetta, P. \&Schemitsch, E.H. (2009). Design, conduct, and interpretation of nonrandomized orthopaedicstudies: a practical approach. (All) evidence matters. The Journal of Bone \& Joint Surgery, 91(Supplement_3), 1-1.

[37] Bryant, D.M., Willits, K. \& Hanson, B.P.( 2009). Principles of designing a cohort study in orthopaedics. The Journal of Bone \& Joint

[38] Surgery, 91(Supplement_3), 10-14.

[39] Kristman, V., Manno, M. \&Côté, P. (2004). Loss to follow-up in cohort studies: how much is too much? European journal of epidemiology, 19(8), 751-760.

[40] Schulz, K.F. \& Grimes, D.A. (2002). Sample size slippages in randomised trials: exclusions and the lost and wayward. The Lancet,

[41] 359(9308), 781-785.

[42] LaValley, M.P., McAlindon, T.E., Chaisson, C.E., Levy, D. \&Felson, D.T. (2001). The validity of different definitions of radiographic worsening for longitudinal studies of knee osteoarthritis. Journal of clinical epidemiology, 54(1), 30-39.

[43] Johnson, R. B., \&Onwuegbuzie, A. J. (2004). Mixed methods research: A research paradigm whose time has come. Educational [44] researcher, 33(7), 14-26.

[45] Li, R. T., Lorenz, S., Xu, Y., Harner, C. D., Fu, F. H., \&Irrgang, J. J. (2011). Predictors of radiographic knee osteoarthritis after anterior cruciate ligament reconstruction. The American Journal of Sports Medicine, 39(12), 2595-2603.

[46] Keays, S. L., Newcombe, P. A., Bullock-Saxton, J. E., Bullock, M. I., \&Keays, A. C. (2010). Factors involved in the development of osteoarthritis after anterior cruciate ligament surgery. The American journal of sports medicine, 38(3), 455-463.

[47] Song, E. K., Seon, J. K., Yim, J. H., Woo, S. H., Seo, H. Y., \& Lee, K. B. (2013). Progression of Osteoarthritis After Double-and

[48] Single- Bundle Anterior Cruciate Ligament Reconstruction. The American journal of sports medicine, 41(10), 2340-2346.

[49] Suomalainen, P., Järvelä, T., Paakkala, A., Kannus, P. \&Järvinen, M.( 2012). Double-bundle versus single- bundle anterior cruciate

[50] ligament reconstruction: a prospective randomized study with 5-year results. The American Journal of Sports Medicine, 40(7), 1511-1518.

[51] Mann, C.J. (2003). Observational research methods. Research design II: cohort, cross sectional, and case- control studies. Emergency Medicine Journal, 20(1), 54-60.

[52] Kellgren, J. H., \& Lawrence, J. S. (1957). Radiological assessment of osteo-arthrosis. Annals of the rheumatic diseases, 16(4), 494502 .

[53] Keays, S.L., Bullock-Saxton, J.E., Keays, A.C., Newcombe, P.A. \& Bullock, M.I. (2007). A 6-year follow- up of the effect of graft site on strength, stability, range of motion, function, and joint degeneration after anterior cruciate ligament reconstruction: patellar tendon versus semitendinosus and Gracilis tendon graft. The American journal of sports medicine, 35(5), 729-739.

[54] Meunier, A., Odensten, M. \& Good, L. (2007). Long-term results after primary repair or non-surgical treatment of anterior cruciate ligament rupture: a randomized study with a 15-year follow-up. Scandinavian journal of medicine \& science in sports, 17(3), 230237.

[55] Higgins, J. P., Green, S., \& Collaboration, C. (2008). Cochrane handbook for systematic reviews of interventions: Wiley Online Library.

[56] Ryś, P., Władysiuk, M., Skrzekowska-Baran, I., \&Małecki, M. T. (2009). and meta-analyses: which can be trusted?.PolskieArchiwum

[57] MedycynyWewnętrznej, 119(3), 148-156.

[58] Gartlehner, G., West, S.L., Mansfield, A.J., Poole, C., Tant, E., Lux, L.J. \&Lohr, K.N. ( 2012). Clinical heterogeneity in systematic reviews and health technology assessments: synthesis of guidance documents and the literature. International Journal of Technology

[59] Assessment in Health Care, 28(1), 36-43.

[60] Lohmander, L.S. \&Roos, H. (1994). Knee ligament injury, surgery and osteoarthritis. Truth or consequences? ActaOrthopaedica, 65(6), 605- 609.

Sami S. "Incidence of Knee Osteoarthritis after Anterior Cruciate Ligament Injury- A Systematic Review." IOSR Journal of Nursing and Health Science (IOSR-JNHS) 6.4 (2017): 46-56. 